INLO-PUB-04/01

\title{
Reflections on Topology - Viewpoints on Abelian Projections *
}

\author{
Pierre van Baal ${ }^{\mathrm{a}}$

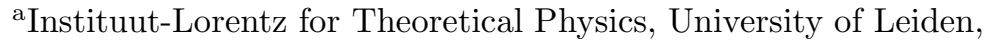 \\ PO Box 9506, NL-2300 RA Leiden, The Netherlands.
}

\begin{abstract}
This talk discusses two topological features in non-abelian gauge theories, related by the notion of abelian projection and the Hopf invariant. Minimising the energy of the non-linear sigma model with a Skyrme-like term (the Faddeev-Niemi model), can be identified with a non-linear maximal abelian gauge fixing of the $S U(2)$ gauge vacua with a winding number equal to the Hopf invariant. In the context of abelian projection the Hopf invariant can also be associated to a monopole world line, through the Taubes winding, measuring its contribution to topological charge. Calorons with non-trivial holonomy provide an explicit realisation. We discuss the identification of its constituent monopoles through degenerate eigenvalues of the Polyakov loop (the singularities or defects of the abelian projection). It allows us to study the correlation between the defect locations and the explicit constituent monopole structure, through a specific $S U(3)$ example.
\end{abstract}

\section{In memory of all those who lost their lifes on 11 September 2001}

\section{Introduction}

Abelian projection was introduced by 't Hooft in an attempt, through a suitable choice of gauge, to decompose a non-abelian gauge field in its neutral and charged components [1]. In its simplest form it involves choosing an observable $X(x)$ that transforms under gauge transformations as $g^{\dagger}(x) X(x) g(x)$, which can be used to diagonalise $X(x)$. This can be done in a smooth way when none of the eigenvalues coincide. The remaining gauge freedom is $U(1)^{r}$, where $r$ is the rank of the gauge group. These are associated with the $r$ neutral gauge bosons in this gauge. Singularities occur when two (or more) eigenvalues coincide, and these can in three dimensions be shown to give rise to (generically) point-like singularities representing magnetic monopoles, as defined with respect to the remnant abelian gauge group.

A smoother, but non-local, abelian gauge fixing can be introduced [1] by taking an abelian field as a background (e.g. for $S U(2)$ the component proportional to $\left.\tau_{3}=\operatorname{diag}(1,-1)\right)$, and imposing the background gauge condition on the charged component of the gauge field. This can be formulated by minimising $\int\left|A_{\mu}^{\mathrm{ch}}(x)\right|^{2}$ along the gauge orbit.

\footnotetext{
*Presented at the workshops "Lattice Hadron Physics", 918 July, Cairns, Australia and "Light-cone Physics: Particles and Strings", 3-11 September 2001, Trento, Italy.
}

Inspired by the abelian projection, Faddeev and Niemi attempted to identify the field $\vec{n}(x)$ (here of unit length, $|\vec{n}(x)|=1$ ) in an $O(3)$ nonlinear sigma model with the local colour direction for $S U(2)$ gauge theory. The hope was that the static knotted solutions constructed numerically 20 in the model originally introduced by Faddeev [3], were possibly related to glueballs [4]. A difficulty is that one would not expect dynamics for a quantity that is associated with the colour direction, due to gauge invariance. Furthermore, the $O(3)$ symmetry is in general spontaneously broken for non-linear sigma models in $3+1$ dimensions. The associated Goldstone bosons are unwanted in non-abelian gauge theories without matter. We will see that nevertheless the $\vec{n}$ field can be identified with an $S U(2)$ gauge field, albeit with zero field strength. Under this identification we have $\left(\partial_{\mu} \vec{n}(x)\right)^{2}=4\left|A_{\mu}^{\text {ch }}(x)\right|^{2}$, giving a hint that minimising the $O(3)$ energy functional can be interpreted [5] in terms of maximal abelian gauge fixing, as will be discussed in section 2 .

The static knotted solutions, as maps $\vec{n}(\vec{x})$ from $S^{3}$ (compactified $R^{3}$ ) to $S^{2}$, are classified by the Hopf invariant. The pre-image of a generic value of $\vec{n}$ traces out a loop in $R^{3}$ (i.e. the collection of points $\vec{x}$, where $\vec{n}(\vec{x})=\vec{n})$. The linking number of any two of such loops is equal to this Hopf invariant. This also coincides with the winding number of the gauge function $g(\vec{x})$, such that 
$n^{a}(\vec{x}) \tau_{a}=g(\vec{x}) \tau_{3} g^{\dagger}(\vec{x})$, with $\tau_{a}$ the Pauli matrices. The associated $S U(2)$ gauge field with zero field strength is $A(\vec{x})=g^{\dagger}(\vec{x}) d g(\vec{x})$.

Such a relation between Hopf invariant and topological charge can also occur for monopoles. A basic monopole is characterised by a hedgehog, with the Higgs field defining the colour direction, pointing out radially. If we gauge rotate the monopole (for the spherically symmetric case, equivalent to a real rotation) while moving along its worldline, a particular point of the hedgehog will trace out a loop whose linking with the monopole worldline is an invariant. This was used by Taubes to make from monopole fields, configurations with non-zero topological charge [6]. In his formulation a monopole-antimonopole pair is created and separated to a finite distance. Kept at this separation one of them is rotated around the axis connecting the two. After this is completed they are brought together and made to annihilate. The time is considered to be euclidean and the rotation introduces a "twist" in the field that prevents the four dimensional configuration from decaying to $A=0$. The obstruction is precisely the topological charge, whose value is equal to the net number of rotations.

This process can just as well be described in terms of a closed monopole loop (in the same way that the Wilson loop is associated with the creation, propagation and annihilation of a heavy quark-antiquark pair), see Fig. 11. The identification with the Hopf invariant should be understood in the following sense: the orientation of the monopole is described by $S U(2) / U(1) \sim S^{2}$ at each point on the monopole loop $\left(S^{1}\right)$, describing a twisted $S^{2}$ bundle over $S^{1}$, e.g. making the total space into an $S^{3}$ for one full "frame" rotation. This is the Hopf fibration, although we have interchanged fibre and base space as compared to the usual formulation.

At finite temperature $A_{0}$ plays the role of the Higgs field and the calorons (periodic instantons) provide an explicit example of this Taubes winding, when it has a non-trivial value of the Polyakov loop at infinity. This Polyakov loop is independent of the directions at infinity, because the finite action of the caloron forces the field strength to vanish at infinity. In this case the caloron splits in $(n$ for $S U(n))$ constituent monopoles, which are the basic spherically symmetric BPS monopoles. Of these, $n-1$ are time independent, whereas the time dependence of the other exactly coincides with the rotation (at a uniform rate) [7,8] of the Taubes winding. In terms of the abelian projection and the introduction of a composite Higgs field the relation of the Hopf invariant with twisted monopole loops has been extensively studied by Jahn [9].

In section 3 we will use the Polyakov loop as the observable $X(\vec{x})$ to be diagonalised, a version of abelian projection that is non-local in time. For the application to the calorons this is not a problem, since at high temperature the action density is static. The static action density is not in conflict with the Taubes winding, as this is associated to a gauge rotation. Defects occur when eigenvalues coincide. Their location is not an artifact of the gauge, since eigenvalues are gauge invariant. But another choice of $X$ will give generically defects at other locations. In the light of this there seems no reason to expect the defects to always be associated to physical lumps. We study in how far this is true in the case where two of the constituents for the $S U(3)$ caloron are brought close together 10].

In section 4 we end with some conclusions concerning the role of topology in non-abelian gauge theories.

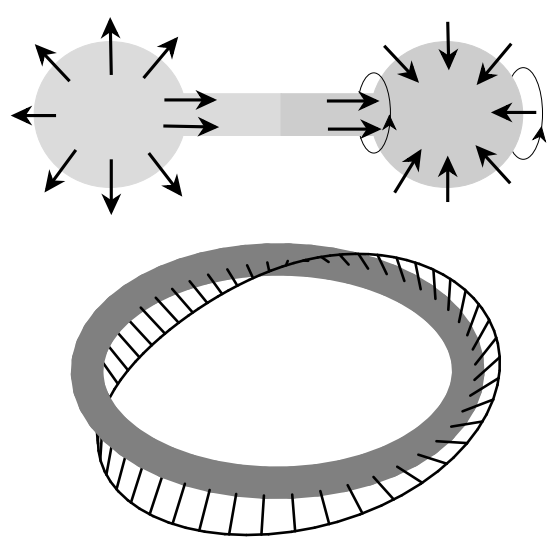

Figure 1. Topological charge constructed from oppositely charged monopoles by rotating one of them. For a closed monopole line, the embedding of the unbroken subgroup makes a full rotation. 


\section{The Faddeev-Niemi model}

The model is defined in terms of a three-vector $\vec{n}(x)$ of fixed (here chosen unit) length. To allow for non-trivial static solutions a Skyrme-like higher-order term is added [3], through the introduction of a composite gauge field strength $F_{\mu \nu}(x)=\frac{1}{2} \vec{n}(x) \cdot\left(\partial_{\mu} \vec{n}(x) \wedge \partial_{\nu} \vec{n}(x)\right)$. A useful identity is $F_{\mu \nu}^{2}(x)=\frac{1}{4}\left(\partial_{\mu} \vec{n}(x) \wedge \partial_{\nu} \vec{n}(x)\right)^{2}$, which follows from the fact that $\partial_{\mu} \vec{n}(x) \wedge \partial_{\nu} \vec{n}(x)=$ $2 F_{\mu \nu}(x) \vec{n}(x)$. Its proportionality to $\vec{n}(x)$ follows from the fact that the latter is perpendicular to $\partial_{\mu} \vec{n}(x)$ (since $\vec{n}(x)$ is a unit three-vector). The action is given up to an overall factor by

$S=\int d^{4} x\left(\partial_{\mu} \vec{n}(x) \cdot \partial^{\mu} \vec{n}(x)-\frac{1}{2} F_{\mu \nu}(x) F^{\mu \nu}(x)\right)$

brought to this simple form by a suitable rescaling of $x$. Finite energy requires $\vec{n}(\vec{x})$ to approach a constant vector at spatial infinity. In this way static configurations are classified by the topological maps from $S^{3}$ into $S^{2}$, characterised by the Hopf invariant. The two-form $F(\vec{x})=\vec{n}(\vec{x}) \cdot(d \vec{n}(\vec{x}) \wedge d \vec{n}(\vec{x}))$ implicitly defines an abelian gauge field one-form $A(\vec{x})$ through $F(\vec{x})=d A(\vec{x})$, in terms of which the Hopf invariant is given by $Q=\frac{1}{4 \pi^{2}} \int A(\vec{x}) \wedge F(\vec{x})$. Remarkably, the energy is bounded by a fractional power of this Hopf invariant 11,12].

$E=\int d^{3} x\left(\left(\partial_{i} \vec{n}(x)\right)^{2}+\frac{1}{2} F_{i j}^{2}(x)\right) \geq c|Q|^{3 / 4}$,

with $c=16 \pi^{2} 3^{3 / 8}$. This gives a rough bound, which can be improved on 13] (by roughly a factor 2). Extensive numerical studies 14,15 have gone up to $Q=8$, with energies indeed following the fractional power of $Q$.

\subsection{Reformulations}

We reformulate the non-linear sigma model in two steps, both well known 16,17]. The first involves $C P_{1}$ fields. Its main advantage is that the abelian gauge field appearing in the Hopf invariant, no longer needs to be defined implicitly. To be specific, one introduces a complex twocomponent field $\Psi(x)$, also having unit length. A further phase is removed by the local abelian gauge invariance, obvious from the following relation to the $n$ field:

$n^{a}(x)=\Psi^{\dagger}(x) \tau^{a} \Psi(x)$.

The abelian gauge invariance of the $C P_{1}$ model leads to a composite gauge field

$A_{\mu}(x)=-i \Psi^{\dagger}(x) \partial_{\mu} \Psi(x)$,

and one verifies that indeed $F(x)=d A(x)$. Useful identities for these computations are the completeness relation $\delta_{i j} \delta_{k l}+\tau_{i j}^{a} \tau_{k l}^{a}=2 \delta_{i l} \delta_{j k}$ and $i \varepsilon_{a b c} \tau_{i j}^{b} \tau_{k l}^{c}=\tau_{k j}^{a} \delta_{i l}-\tau_{i l}^{a} \delta_{j k}$. The energy, Eq. (2), becomes

$E=\int d^{3} x\left(4\left|D_{i} \Psi(\vec{x})\right|^{2}-\frac{1}{2} F_{i j}^{2}(\vec{x})\right)$,

with $D_{\mu}=\partial_{\mu}-i A_{\mu}(x)$ the covariant derivative.

Next we make use of the fact that any complex two-component vector of unit length is in one to one relation to an $S U(2)$ group element. Alternatively we can write $\Psi(x)=g(x) \Psi_{0}$. For convenience we choose $\Psi_{0}^{\dagger}=(1,0)$, such that

$n_{a}(x)=\frac{1}{2} \operatorname{tr}\left(\tau_{3} g^{\dagger}(x) \tau_{a} g(x)\right)$.

We introduce $J_{\mu}(x) \equiv i \tau_{a} J_{\mu}^{a}(x) \equiv g^{\dagger}(x) \partial_{\mu} g(x)$, which can be interpreted as the components of an $S U(2)$ gauge connection with vanishing curvature, that is $G(x)=d J(x)+J(x) \wedge J(x)=0$, where $J(x) \equiv J_{\mu}(x) d x_{\mu}$. A simple calculation shows that $A_{\mu}(x)=J_{\mu}^{3}(x)$ and $\partial_{\mu} \Psi^{\dagger}(x) \partial^{\mu} \Psi(x)=$ $J_{\mu}^{a}(x) J_{a}^{\mu}(x)$. The zero non-abelian field strength leads to $F_{\mu \nu}(x)=2\left(J_{\mu}^{1}(x) J_{\nu}^{2}(x)-J_{\nu}^{1}(x) J_{\mu}^{2}(x)\right)$, or

$F(x)=d J^{3}(x)=2 J^{1}(x) \wedge J^{2}(x)$,

in terms of $J^{a}(x) \equiv J_{\mu}^{a}(x) d x_{\mu}$. This implies that $A(\vec{x}) \wedge F(\vec{x})=2 J^{3}(\vec{x}) \wedge J^{1}(\vec{x}) \wedge J^{2}(\vec{x})$, and substituting $J(x)=g^{\dagger}(x) d g(x)$ one finds that the Hopf invariant is exactly equal [14] to the winding number of the gauge function $g(\vec{x})$,

$\frac{1}{4 \pi^{2}} A(\vec{x}) \wedge F(\vec{x})=\frac{1}{24 \pi^{2}} \operatorname{tr}\left(g^{\dagger}(\vec{x}) d g(\vec{x})\right)^{3}$.

Using that $J$ has zero curvature, we can of course also relate the Hopf invariant to the non-abelian Chern-Simons form, giving $\frac{1}{4 \pi^{2}} A(\vec{x}) \wedge F(\vec{x})=$ $-\frac{1}{8 \pi^{2}} \operatorname{tr}\left[J(\vec{x}) \wedge\left(d J(\vec{x})+\frac{2}{3} J(\vec{x}) \wedge J(\vec{x})\right)\right] \equiv C S(J)$. 


\subsection{The gauge fixing interpretation}

The natural question to ask now, is what the interpretation of the energy functional becomes in terms of the non-abelian gauge field

$E=\int d^{3} x\left(4\left(J_{i}^{\alpha}(\vec{x})\right)^{2}+2\left(\epsilon_{\alpha \beta} J_{i}^{\alpha}(\vec{x}) J_{j}^{\beta}(\vec{x})\right)^{2}\right)$,

where the index $\alpha$ and $\beta$ run only over 1 and 2 . With the absence of the neutral component, $J_{\mu}^{3}(x)$, the $S U(2) / U(1)$ formulation is evident. It defines a positive definite functional on the gauge orbit, and its minimum can thus be seen as a particular (non-linear) maximal abelian gauge fixing, leaving the abelian subgroup generated by $\tau_{3}$ unfixed [1, 18]. As the reparametrisation is mathematically equivalent, we are entitled to interpret the minima of the energy functional in the sector with a given value of $Q$ as gauge fixed pure gauge (i.e. curvature free, or flat) connections in a sector with gauge field winding number $Q$. Therefore, there is a gauge fixing in terms of which the gauge vacua with different winding number can be characterised by inequivalent knots. We thus conclude that instantons "knit", interpolating between different types of "knots".

The relation between $\vec{n}$ and flat $S U(2)$ gauge fields can also be understood from geometric quantisation on $S U(2) / U(1) \sim S^{2}$ and chiral models, but the relation to gauge fixing was only noted recently [5]. Finding the absolute minimum of a gauge fixing functional is known to be a hard problem, seemingly reflected in the difficulty of finding the knots with the lowest energy for a given value of $Q$, as clearly illustrated in Fig. 2 of Ref. 115

\subsection{Implications}

Much work has been invested in interpreting this model as an effective low-energy representation of $S U(2)$ gauge theory [ [ $, 19,20$. The quality of this approximation is being investigating by inverse Monte Carlo techniques 21. Amongst other things, these numerical results have reported the presence of the unwanted massless Goldstone bosons, if one does not include terms that explicitly break the $O(3)$ symmetry. Also a perturbative renormalisation group study showed there are difficulties with the restricted formula- tion of the model, as an effective description of $S U(2)$ gauge theory 22]. As we will argue, this difficulty seems to be related to the fact that one expands around a background that is actually unstable.

The conventional starting point in these studies is based on the decomposition [23]

$\vec{A}_{\mu}(x)=\partial_{\mu} \vec{n}(x) \wedge \vec{n}(x)+C_{\mu}(x) \vec{n}(x)+\vec{W}_{\mu}(x),(10)$

where $C_{\mu}$ describes an abelian gauge field and $W_{\mu}^{a}$ are the charged components of the field, with respect to $n^{a}(x) \tau_{a}$. One attempts to integrate out both $W_{\mu}^{a}$ and, unlike in abelian projection, $C_{\mu}$. In perturbation theory this amounts to a background field calculation. For later use we consider the one parameter family of backgrounds $\vec{A}_{\mu}(x)=q \partial_{\mu} \vec{n}(x) \wedge \vec{n}(x)$. For $q=1 \vec{n}(x)$ is covariantly constant, i.e. $\partial_{\mu} \vec{n}(x)+\vec{A}_{\mu}(x) \wedge \vec{n}(x)=0$, which remains true when adding $C_{\mu} \vec{n}$ to the background. It ensures that the abelian gauge field transforms properly under gauge transformations after abelian projection 23. To address the issue of perturbative stability, we compute the field strength for general $q$,

$\vec{F}_{\mu \nu}(\vec{x})=q(q-2) \partial_{\mu} \vec{n}(\vec{x}) \wedge \partial_{\nu} \vec{n}(x)$,

which is non-zero for $q=1$. The energy of this background field is proportional to $q^{2}(2-q)^{2}$ and actually has a local maximum at $q=1$, and hence is unstable. On the other hand, the energy of the background vanishes not only for $q=0$ (the trivial background), but also for $q=2$. The latter corresponds to the flat connection $J$ that has topological charge equal to $Q$, given by the integral of the non-abelian Chern-Simons form over space. Rescaling the flat connection $J$ with $q / 2$ one easily finds its value for any $q$, $\int C S(q J(\vec{x}) / 2)=q^{2}(3-q) Q / 4$. For $q=1$, this relation between the Hopf invariant and the nonabelian Chern-Simons form can also be found in Ref. [19]. We note that the integral gives half the Hopf invariant for $q=1$, thus in some sense this background is "half-way" between two vacua.

For $q=2$ the (flat) background is useful in separating the gauge field in its different topological sectors, since small fluctuations parametrised by $C_{\mu}$ and $W_{\mu}^{a}$ do not change the value of the 
winding number. With what we could call the Faddeev-Niemi gauge fixing, such a separation in different topological sectors is now possible in localised form, without having to appeal to the asymptotics of the gauge field. This may lead to new tools and insights to deal with the non-trivial topology of non-abelian gauge theories.

\section{The SU(3) Caloron}

We now turn our attention to finite temperature instantons (calorons) with a Polyakov loop, $P(\vec{x})=P \exp \left(\int_{0}^{\beta} A_{0}(\vec{x}, t) d t\right)$ in the periodic gauge $A_{\mu}(t, \vec{x})=A_{\mu}(t+\beta, \vec{x})$, non-trivial at spatial infinity (specifying the holonomy). It implies the spontaneous breakdown of gauge symmetry. For a charge one $S U(n)$ caloron, their are $n$ constituent monopoles [8]. Locations of constituents monopoles can be identified through:

- Points where two eigenvalues of the Polyakov loop coincide, which is where the $U^{n-1}(1)$ symmetry is partially restored to $S U(2) \times U^{n-2}(1)$.

- The centers of mass of the (spherical) lumps.

- The Dirac monopoles (or rather dyons, due to self-duality) as the sources of the abelian field lines, extrapolated back to the cores.

If well separated and localised, all these coincide [7,24]. This is no longer the case when two constituents come close together, as shown for $S U(2)$ in Ref. [25], and for $S U(3)$ in Ref. [10].

The locations of the constituent monopoles may be chosen at will, but their masses are fixed by the eigenvalues of $\mathcal{P}_{\infty} \equiv \lim _{|\vec{x}| \rightarrow \infty} P(\vec{x})$. These eigenvalues can be ordered by a constant gauge transformation $W_{\infty}$

$$
\begin{aligned}
& W_{\infty}^{\dagger} \mathcal{P}_{\infty} W_{\infty}=\mathcal{P}_{\infty}^{0}=\exp \left[2 \pi i \operatorname{diag}\left(\mu_{1}, \ldots, \mu_{n}\right)\right] \\
& \mu_{1} \leq \ldots \leq \mu_{n} \leq \mu_{n+1} \equiv 1+\mu_{1},
\end{aligned}
$$

with $\sum_{m=1}^{n} \mu_{m}=0$. Using the classical scale invariance to put the extent of the euclidean time direction to one, $\beta=1$, the masses of the constituent monopoles are now given by $8 \pi^{2} \nu_{i}$, where $\nu_{i} \equiv \mu_{i+1}-\mu_{i}$.

Similarly we can bring $P(\vec{x})$ to the diagonal form, with eigenvalues ordered on the circle, by a local gauge function, $P(\vec{x})=W(\vec{x}) P_{0}(\vec{x}) W^{\dagger}(\vec{x})$. We note that $W(\vec{x})$ (unique up to a residual abelian gauge rotation) and $P_{0}(\vec{x})$ will be smooth, except where two (or more) eigenvalues coincide. The ordering shows there are $n$ different types of singularities (called defects [26]), for each of the neighbouring eigenvalues to coincide. The first $n-1$ are associated with the basic monopoles (as part of the inequivalent $S U(2)$ subgroups related to the generators of the Cartan subgroup). The $n^{\text {th }}$ defect arises when the first and the last eigenvalue (still neighbours on the circle) coincide. Its magnetic charge ensures charge neutrality of the caloron, and it carries the Taubes winding supporting the non-zero topological charge [7,8,27].

The topological charge can be reduced to surface integrals near the singularities with the use of $\operatorname{tr}\left(P^{\dagger} d P\right)^{3}=d 3 \operatorname{tr}\left(\left(P_{0}^{\dagger} A_{W} P_{0}+2 P_{0}^{\dagger} d P_{0}\right) \wedge A_{W}\right)=$ $d 3 \operatorname{tr}\left(A_{W} \wedge\left(2 A_{W} \log P_{0}+P_{0} A_{W} P_{0}^{\dagger}\right)\right)$, where $A_{W} \equiv$ $W^{\dagger} d W$. If one assumes all defects are pointlike, this can be used to show that for each of the $n$ types the (net) number of defects has to equal the topological charge, the type being selected by the branch of the logarithm (associated with the $n$ elements in the center) [26]. This is the generic situation, but in special cases defects may form a submanifold, as we will find for a global embedding of the $S U(2)$ caloron in $S U(3)$.

\subsection{Coinciding constituents}

One might expect defects to merge when constituent monopoles do. The resulting triple degeneracy of eigenvalues for $S U(3)$ implies that the Polyakov loop takes on a value in the center. Yet this can be shown not to occur for the $S U(3)$ caloron with unequal masses. We therefore seem to have (at least) one more defect than the number of constituents, when two merging constituents will manifest themselves as one constituent. To study what happens in this case we first recall the simple formula for the $S U(n)$ action density [8]

$$
\begin{aligned}
& \operatorname{Tr} F_{\mu \nu}^{2}(x)=\partial_{\mu}^{2} \partial_{\nu}^{2} \log \left[\frac{1}{2} \operatorname{tr}\left(\mathcal{A}_{n} \cdots \mathcal{A}_{1}\right)-\cos (2 \pi t)\right], \\
& \mathcal{A}_{m} \equiv \frac{1}{r_{m}}\left(\begin{array}{cc}
r_{m} & \left|\vec{y}_{m}-\vec{y}_{m+1}\right| \\
0 & r_{m+1}
\end{array}\right)\left(\begin{array}{cc}
c_{m} & s_{m} \\
s_{m} & c_{m}
\end{array}\right),
\end{aligned}
$$

with $\vec{y}_{m}$ the center of mass location of the $m^{\text {th }}$ constituent monopole. We defined $r_{m} \equiv\left|\vec{x}-\vec{y}_{m}\right|$, $c_{m} \equiv \cosh \left(2 \pi \nu_{m} r_{m}\right), s_{m} \equiv \sinh \left(2 \pi \nu_{m} r_{m}\right)$, as well as $\vec{y}_{n+1} \equiv \vec{y}_{1}, r_{n+1} \equiv r_{1}$. We are interested in the 
case where the problem of two coinciding constituents in $S U(n)$ is mapped to the $S U(n-1)$ caloron. For this we restrict to the case where $\vec{y}_{m}=\vec{y}_{m+1}$ for some $m$, which for $S U(3)$ is always the case when two constituents coincide. Since now $r_{m}=r_{m+1}$, one easily verifies that $\mathcal{A}_{m+1} \mathcal{A}_{m}=\mathcal{A}_{m+1}\left[\nu_{m+1} \rightarrow \nu_{m}+\nu_{m+1}\right]$, describing a single constituent monopole (with properly combined mass), reducing Eq. (13) to the $S U(n-1)$ action density, with $n-1$ constituents.

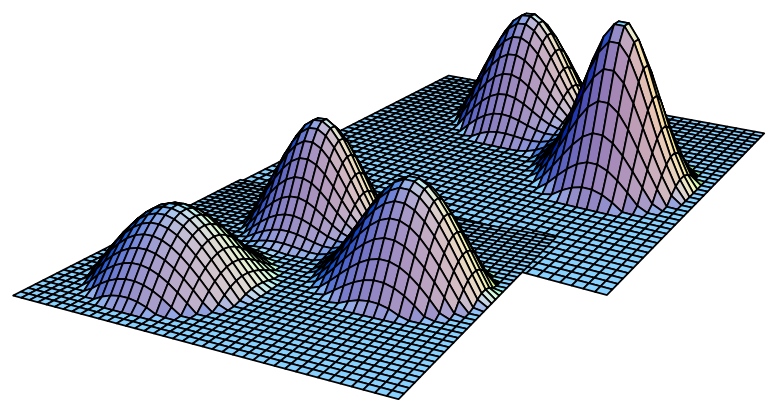

Figure 2. Action densities for $S U(3)$ calorons on equal logarithmic scales, cut off at $\frac{1}{2} e^{-1}$, for $\beta=1$ in the plane defined by the constituent locations $\vec{y}_{1}=(-2,-2,0), \vec{y}_{2}=(0,2,0), \vec{y}_{3}=(2,-1,0)$ and $t=0$, using $\left(\mu_{1}, \mu_{2}, \mu_{3}\right)=(-17,-2,19) / 60$. On the right we moved the first constituent to the location of the second.

\subsection{Tracing the defects}

We will study in detail a generic example in $S U(3)$, with $\left(\mu_{1}, \mu_{2}, \mu_{3}\right)=(-17,-2,19) / 60$. Fig. 2 shows the action density both for noncoinciding constituents and when moving the first constituent to the location of the second. We denote by $\vec{z}_{m}$ the position associated with the $m^{\text {th }}$ constituent, where two eigenvalues of the Polyakov loop coincide (the defect locations). Numerically it was established that 24, in the gauge where $\mathcal{P}_{\infty}=\mathcal{P}_{\infty}^{0}$ (see Eq. (12)),

$$
\begin{aligned}
& P_{1}=P\left(\vec{z}_{1}\right)=\operatorname{diag}\left(e^{-\pi i \mu_{3}}, \quad e^{-\pi i \mu_{3}}, \quad e^{2 \pi i \mu_{3}}\right), \\
& P_{2}=P\left(\vec{z}_{2}\right)=\operatorname{diag}\left(e^{2 \pi i \mu_{1}}, \quad e^{-\pi i \mu_{1}}, e^{-\pi i \mu_{1}}\right), \\
& P_{3}=P\left(\vec{z}_{3}\right)=\operatorname{diag}\left(-e^{-\pi i \mu_{2}}, \quad e^{2 \pi i \mu_{2}},-e^{-\pi i \mu_{2}}\right) .
\end{aligned}
$$

This is for any choice of holonomy and constituent locations (with the proviso they are well sepa- rated, i.e. their cores do not overlap, in which case to a good approximation $\left.\vec{z}_{m}=\vec{y}_{m}\right)$. Now we take $\vec{y}_{1}=(0,0,10+d), \vec{y}_{2}=(0,0,10-d)$ and $\vec{y}_{3}=(0,0,-10)$. The limit of coinciding constituents is achieved by $d \rightarrow 0$. In very good approximation, as long as the first two constituents remain well separated from the third constituent (carrying the Taubes winding), $P_{3}$ will be constant in $d$ and the $S U(3)$ gauge field 24] of the first two constituents will be constant in time (in the periodic gauge). Thus $P\left(\vec{z}_{m}\right)=\exp \left(A_{0}\left(\vec{z}_{m}\right)\right)$ for $m=1,2$. This simplifies the calculation of the Polyakov loop considerably.

When the cores of the two approaching constituents start to overlap, $P_{1}$ and $P_{2}$ are no longer diagonal (mixing the lower $2 \times 2$ components). At $d=0$ they are diagonal again, but $P_{2}$ will be no longer in the fundamental Weyl chamber (the "logarithm" of the Cartan subgroup). A Weyl reflection maps it back, while for $d \neq 0$ a more general gauge rotation back to the Cartan subgroup is required to do so, see Fig. 3. For $d \rightarrow 0$, each $P_{m}$ (and $\mathcal{P}_{\infty}$ ) falls on the dashed line, defining the reduction to $S U(2)$.

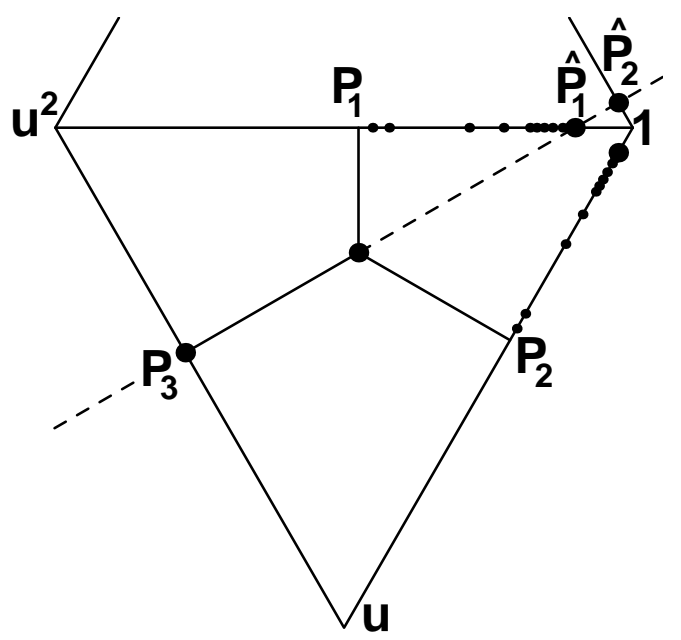

Figure 3. The fundamental Weyl chamber with the positions of $P_{m}$ indicated at $d=2,1, .2, .1$, $.05, .04, .03, .02, .01, .005, .001, .0005$, and (large dots) 0 . The perpendiculars point to $\mathcal{P}_{\infty}$ (center), and emanate from the values of $P_{m}$ for well separated constituents. The dashed line shows the $S U(2)$ embedding for $d=0 .(u \equiv \exp (2 \pi i / 3) \mathbf{1})$ 
To illustrate this more clearly, we give the expressions for $P_{m}$ (which we believe to hold for any non-degenerate choice of the $\mu_{i}$ ) when $d \rightarrow 0$ :

$$
\begin{aligned}
& \hat{P}_{1}=P\left(\vec{z}_{1}\right)=\operatorname{diag}\left(e^{2 \pi i \mu_{2}}, \quad e^{2 \pi i \mu_{2}}, \quad e^{-4 \pi i \mu_{2}}\right), \\
& \hat{P}_{2}=P\left(\vec{z}_{2}\right)=\operatorname{diag}\left(e^{-\pi i \mu_{2}}, \quad e^{2 \pi i \mu_{2}}, e^{-\pi i \mu_{2}}\right),(15) \\
& \hat{P}_{3}=P\left(\vec{z}_{3}\right)=\operatorname{diag}\left(-e^{-\pi i \mu_{2}}, \quad e^{2 \pi i \mu_{2}},-e^{-\pi i \mu_{2}}\right) \text {. }
\end{aligned}
$$

These can be factorised as $\hat{P}_{m}=\hat{P}_{2} Q_{m}$, where $\hat{P}_{2}$ describes an overall $U(1)$ factor. In terms of $Q_{1}=\operatorname{diag}\left(e^{3 \pi i \mu_{2}}, 1, e^{-3 \pi i \mu_{2}}\right), Q_{2}=\operatorname{diag}(1,1,1)=\mathbf{1}$ and $Q_{3}=\operatorname{diag}(-1,1,-1)$ the $S U(2)$ embedding in $S U(3)$ becomes obvious. It leads for $Q_{2}$ to the trivial and for $Q_{3}$ to the non-trivial element of the center of $S U(2)$ (appropriate for the latter, carrying the Taubes winding). On the other hand, $Q_{1}$ corresponds to $\operatorname{diag}\left(e^{3 \pi i \mu_{2}}, e^{-3 \pi i \mu_{2}}\right)$, which for the $S U(2)$ caloron is not related to coinciding eigenvalues. For $d \rightarrow 0$, Fig. $\theta$ shows that $\vec{z}_{1}$ gets "stuck" at a finite distance (0.131419) from $\vec{z}_{2}$.

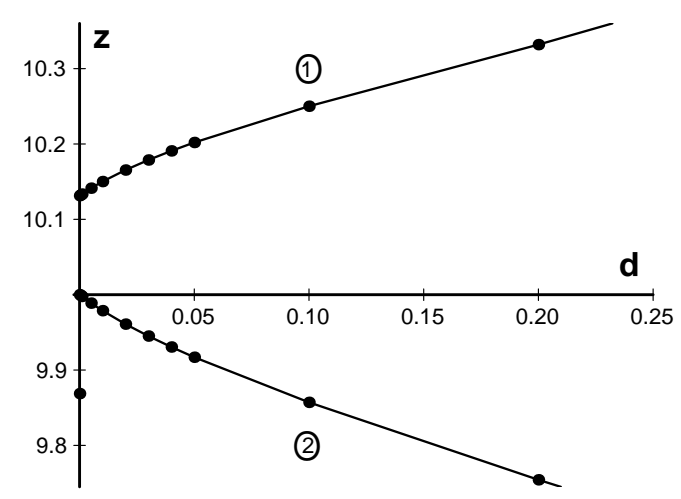

Figure 4 . The defect locations $\vec{z}_{1}$ and $\vec{z}_{2}$, along the $z$-axis, for $M \equiv \nu_{2}-\nu_{1}=0.1$ as a function of $d$.

\subsection{Spurious defects}

The $S U(2)$ embedding determines the caloron solution for $d=0$, with constituent locations $\vec{y}_{1}^{\prime}=$ $\vec{y}_{2}$ and $\vec{y}_{2}^{\prime}=\vec{y}_{3}$, and masses $\nu_{1}^{\prime}=\nu_{1}+\nu_{2}=\mu_{3}-\mu_{1}$ and $\nu_{2}^{\prime}=\nu_{3}$. The spurious nature of the defect is obvious by calculating its location purely in terms of this $S U(2)$ caloron, demanding the $S U(2)$ Polyakov loop to equal $\operatorname{diag}\left(e^{3 \pi i \mu_{2}}, e^{-3 \pi i \mu_{2}}\right)$. For this we can use the analytic expression [25] of the $S U(2)$ Polyakov loop along the $z$-axis. The location of the spurious defect, $\vec{z}_{1}=(0,0, z)$, is found by solving $3 \pi \mu_{2}=\pi \nu_{2}^{\prime}-\frac{1}{2} \partial_{z} \operatorname{acosh}\left[\frac{1}{2} \operatorname{tr}\left(\mathcal{A}_{2}^{\prime} \mathcal{A}_{1}^{\prime}\right)\right]$. For our example, $z=10.131419$ indeed verifies this equation.

Fig. . gives the spurious location as a function of the mass difference of the two coinciding constituents. We find that only when this difference vanishes, the defects merge to form a triple degeneracy. Using the relation $3 \mu_{2}=\nu_{1}-\nu_{2}$, the case of equal masses for the coinciding constituents corresponds to $\mu_{2}=0$. For unequal masses the defect is always spurious, but it tends to stay within reach of the non-abelian core of the coinciding constituent monopoles, $\left(\pi \nu_{1}^{\prime}\right)^{-1} \sim 0.53$, except when the mass difference approaches its extremal values $\pm \nu_{1}^{\prime}= \pm\left(1-\nu_{3}\right)$. At these extremal values one of the $S U(3)$ constituents becomes massless and delocalised, which we excluded for $d \neq 0$.

Actually, the limit $d \rightarrow 0$ is singular due to the global decomposition into $S U(2) \times U(1)$ at $d=0$. Gauge rotations $U$ in the global $S U(2)$ subgroup do not affect $\hat{P}_{2}$, and therefore any $U Q_{1} U^{\dagger}$ gives rise to the same accidental degeneracy. In particular solving $-3 \pi \mu_{2}=\pi \nu_{2}^{\prime}-\frac{1}{2} \partial_{z} \operatorname{acosh}\left[\frac{1}{2} \operatorname{tr}\left(\mathcal{A}_{2}^{\prime} \mathcal{A}_{1}^{\prime}\right)\right]$ (corresponding to the Weyl reflection $Q_{1} \rightarrow Q_{1}^{\dagger}$ ) yields $z=9.868757$ for $\mu_{2}=-1 / 30$ (isolated point in Fig. (4). Indeed, $U \in S U(2) / U(1)$ traces out a (nearly spherical) shell where two eigenvalues of $P$ coincide (note that for $\mu_{2}=0$ this shell collapse to a single point, $z=10$ ). A perturbation tends to remove this accidental degeneracy.

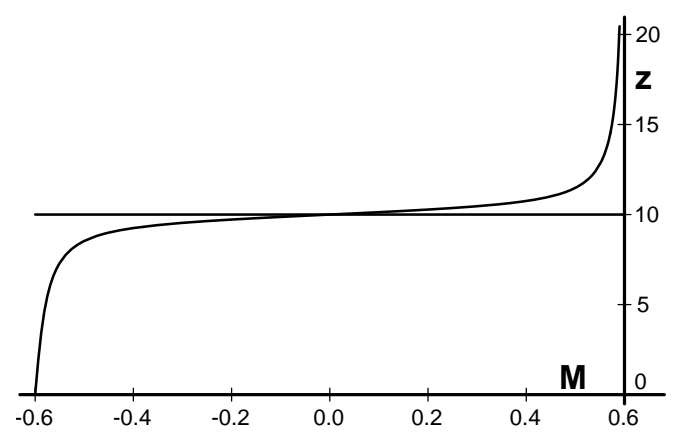

Figure 5. The defect locations $\vec{z}_{1}$ and $\vec{z}_{2}$, along the $z$-axis, as a function of $M \equiv \nu_{2}-\nu_{1}$ for $d \rightarrow 0$. 


\section{Conclusions}

Topology is important for the non-perturbative understanding of non-abelian gauge theories, because it forces one to take serious that the configuration space is non-trivial [28]. That this has a role to play in the quantum theory can be easily understood from the difference between quantisation on the line versus the circle.

Attempts to localise topology by gauge fixing (and its defects), should always be treated with healthy suspicion. This is illustrated by our interpretation of the knotted solitons in the FaddeevNiemi model in terms of a non-linear maximal abelian gauge fixing of the gauge vacuum: There is nothing physical to be localised in a classical vacuum. Also we have shown that the notion of abelian projected monopoles has to be considered with care. For "smooth" configurations and "reasonable" choices of projections defects may well be correlated to action lumps, but this is by no means guaranteed. Furthermore, the defects can at best serve as bare quantities, which need to be dressed by the quantum corrections.

What is perhaps more important is that we learn that instantons and monopoles are closely connected, and should not be viewed in isolation. In disordered field configurations, gauge dislocations are likely to occur [28]. In four dimensions these are instantons (point dislocations), monopoles (line dislocations) and vortices (surface dislocations). Even when there is a (sufficient) separation of scales, the problem remains how to isolate the effective low energy degrees of freedom in a reliable manner.

\section{Acknowledgements}

The first half of this talk is based on joint work with Andreas Wipf. I thank him for collaboration, as well as for a provocative question that formed the inspiration for the the second half. I also thank Chris Ford for discussions and pointing out that the $S U(3)$ embedding of the $S U(2)$ caloron should give rise to defects forming a surface, rather than a point. Discussions with Poul Damgaard, Leander Dittmann, Gerald Dunne, Ludwig Faddeev, Thomas Heinzl,
Marek Karliner, Martin Lüscher, Holger Gies, Parameswaran Nair and Joaquin Sanchez Guillen are gratefully acknowledged, as well as hospitality at Cern. Finally I thank the organisers of the workshops in Cairns and Trento for their kind and generous invitations.

\section{REFERENCES}

1. G. 't Hooft, Nucl. Phys. B 190 (1981) 455.

2. L. Faddeev and A. J. Niemi, Nature 387 (1997) 58 hep-th/9610193.

3. L. D. Faddeev, "Quantization of Solitons," Print-75-0570 (IAS, Princeton).

4. L. Faddeev and A. J. Niemi, Phys. Rev. Lett. 82 (1999) 1624 hep-th/9807069.

5. P. van Baal and A. Wipf, Phys. Lett. B 515 (2001) 181 hep-th/0105141.

6. C. Taubes, in: Progress in gauge field theory, eds. G.'t Hooft e.a., (Plenum Press, New York, 1984) p.563.

7. T. C. Kraan and P. van Baal, Nucl. Phys. B 533 (1998) 627 hep-th/9805168.

8. T. C. Kraan and P. van Baal, Phys. Lett. B 435 (1998) 389 hep-th/9806034.

9. O. Jahn, J. Phys. A 33 (2000) 2997 hepth/9909004.

10. P. van Baal, "Abelian projected monopoles to be or not to be," hep-lat/0108027.

11. A. F. Vakulenko and L. V. Kapitansky, Sov. Phys. Dokl. 24 (1979) 433.

12. A. Kundu and Y. P. Rybakov, J. Phys. A 15 (1982) 269.

13. R. S. Ward, Nonlinearity 12 (1999) 1 hepth/9811176.

14. R. A. Battye and P. M. Sutcliffe, Phys. Rev. Lett. 81 (1998) 4798 hep-th/9808129]; Proc. Roy. Soc. Lond. A 455 (1999) 4305 hepth/9811077].

15. J. Hietarinta and P. Salo, Phys. Rev. D 62 (2000) 081701.

16. H. Eichenherr, Nucl. Phys. B 146 (1978) 215 [Erratum-ibid. B 155 (1978) 544].

17. A. D'Adda, M. Lüscher and P. Di Vecchia, Nucl. Phys. B 146 (1978) 63.

18. A. S. Kronfeld, M. L. Laursen, G. Schierholz and U. J. Wiese, Phys. Lett. B 198 (1987) 516. 
19. E. Langmann and A. J. Niemi, Phys. Lett. B 463 (1999) 252 hep-th/9905147.

20. Y. M. Cho, H. Lee and D. G. Pak, "Effective theory of QCD," hep-th/9905215.

21. L. Dittmann, T. Heinzl and A. Wipf, poster at Lattice 2001, Berlin, July 2001, to appear.

22. H. Gies, Phys. Rev. D 63 (2001) 125023 hepth/0102026.

23. Y. M. Cho, Phys. Rev. D 21 (1980) 1080.

24. P. van Baal, in: Lattice fermions and structure of the vacuum, eds. V.Mitrjushkin and G. Schierholz (Kluwer, Dordrecht, 2000) p. 269 hep-th/9912035.
25. M.García Pérez, A.González-Arroyo, A. Montero and P. van Baal, JHEP 9906 (1999) 001 hep-lat/9903022.

26. C. Ford, T. Tok and A. Wipf, Nucl. Phys. B 548 (1999) 585 hep-th/9809209]; Phys. Lett. B 456 (1999) 155 hep-th/9811248.

27. M. N. Chernodub, T. C. Kraan and P. van Baal, Nucl. Phys. Proc. Suppl. 83 (2000) 556 hep-lat/9907001.

28. P. van Baal, in: At the Frontiers of Particle Physics - Handbook of QCD, Vol.2, ed. M. Shifman (World Scientific, Singapore, 2001), pp. 683-760 hep-ph/0008206]. 